\title{
Sanguinarine induces apoptosis of human lens epithelial cells by increasing reactive oxygen species via the MAPK signaling pathway
}

\author{
YUE ZHANG ${ }^{*}$ and WAN-RONG HUANG ${ }^{*}$
}

Tianjin Eye Hospital, Clinical College of Ophthalmology, Tianjin Medical University, Tianjin 300020, P.R. China

Received March 11, 2018; Accepted February 21, 2019

DOI: $10.3892 / \mathrm{mmr} .2019 .10087$

\begin{abstract}
Posterior capsular opacification (PCO) remains a major complication of cataract surgery and is the most common reason for loss of vision. PCO is primarily associated with uncontrolled proliferation of residual human lens epithelial cells (HLEs). Sanguinarine is a type of benzophenanthridine alkaloid extracted from the herbaceous plant Sanguinaria canadensis, which is widely used for its anti-microbial, anti-inflammatory, anti-oxidative and anti-proliferative properties. However, studies examining the effect of sanguinarine on HLEs and the underlying mechanism are scarce. The present study aimed to investigate the effects of sanguinarine on HLEs. An MTT assay was used to determine the effect of sanguinarine on cell viability. Flow cytometry was used to evaluate cell apoptosis, and the mitochondrial membrane potential and reactive oxygen species (ROS) levels. A caspase $3 / 7$ activity assay was also used to evaluate cell apoptosis, while western blotting was performed to determine protein levels. The results demonstrated that sanguinarine exerted an anti-proliferative effect by inducing ROS, and caused cell apoptosis via mitochondrial and caspase-dependent pathways. Treatment with sanguinarine led to the loss of mitochondrial membrane potential. Sanguinarine also significantly increased the phosphorylation levels of c-Jun N-terminal kinase and p38, which indicated the involvement of the mitogen-activated protein kinase signaling pathway. These results suggested that sanguinarine may have a noteworthy pro-apoptotic effect on HLEs, and may be used as a potential drug for PCO or even cataract prevention.
\end{abstract}

Correspondence to: Dr Wan-Rong Huang, Tianjin Eye Hospital, Clinical College of Ophthalmology, Tianjin Medical University, 4 Gansu Road, Tianjin 300020, P.R. China

E-mail: scarlethwang@aliyun.com

${ }^{*}$ Contributed equally

Key words: sanguinarine, posterior capsular opacification, human lens epithelial cells, apoptosis

\section{Introduction}

Posterior capsule opacification (PCO) and impaired vision are complications associated with cataract surgery, and the high complication rate is due to regeneration of residual lens epithelial cells (1). PCO remains a major complication of cataract surgery and the most common cause of vision loss (2). Despite current advances in PCO research, including basic research on cataracts, the development of surgical techniques and the development of materials used in intraocular lenses, PCO prevalence is reported to be close to $100 \%$ in children and $30-40 \%$ in adults within 5 years post-surgery (3-5). Pharmacological drugs and small molecules have been used to prevent $\mathrm{PCO}$ in recent years, but their efficiency is poor and they may cause serious side effects (6). Therefore, it is necessary to develop novel drugs that may effectively inhibit the proliferation of HLEs or prevent PCO.

Sanguinarine is a type of benzophenanthridine alkaloid extracted from the roots of the herbaceous plant Sanguinaria canadensis (7), which is widely used for its anti-microbial, anti-inflammatory, anti-oxidative and tumor-suppressing properties (8-12). Recent studies have indicated that sanguinarine is able to suppress inflammation by reducing the expression of inflammatory cytokines, including tumor necrosis factor- $\alpha$ and interleukins $1 \beta$ and 6 in rats (13). Moreover, sanguinarine has been reported to exhibit anti-tumorigenic activity by promoting apoptosis in acute lymphoblastic leukemia (ALL), basal-like breast cancer and colorectal cancer (14-16). Treatment of ALL cells with sanguinarine was reported to cause apoptosis through loss of mitochondrial membrane potential (MMP), activation of caspase pathways and generation of reactive oxygen species (ROS) (15). Furthermore, sanguinarine-induced apoptosis is strongly associated with multiple signaling pathways including the mitogen-activated protein kinase (MAPK) (17), phosphoinositide-3-kinase/protein kinase B (18), Wnt/ $\beta$-catenin (19) and Toll-like receptor 4/nuclear factor- $\kappa \mathrm{B}$ signaling pathways (20). However, to the best of our knowledge, few studies have focused on examining its efficacy on HLEs and its underlying mechanism.

The purpose of the present study was to understand the effects of sanguinarine on HLEs and the its underlying mechanisms of action. Firstly, the potential anti-proliferative effects of sanguinarine on HLEs were examined. Subsequently, 
the induction of apoptosis or autophagy was verified to be the mechanism underlying the anti-proliferative effect of sanguinarine. In addition, the present study further assessed whether sanguinarine-induced apoptosis was achieved via mitochondrial and caspase-dependent pathways, whether ROS generation served a role in this process and whether MAPK signaling was also involved in human lens epithelial (HLE) cell death following treatment with sanguinarine.

\section{Materials and methods}

Chemicals. Sanguinarine powder, dimethyl sulfoxide (DMSO) and the ROS inhibitor N-acetylcysteine (NAC) were obtained from Sigma-Aldrich (Merck KGaA, Darmstadt, Germany). Antibodies against B-cell lymphoma 2 (Bcl-2) and apoptosis regulator BAX (Bax) were purchased from Santa Cruz Biotechnology, Inc. (Dallas, TX, USA), while antibodies against p38, c-Jun N-terminal kinase (JNK), phosphorylated (phospho)-JNK, phospho-p38 and $\beta$-actin were purchased from Cell Signaling Technology Inc. (Danvers, MA, USA). The pan-caspase inhibitor benzyloxycarbonyl-Val-Ala-Asp (OMe) fluoromethylketone (z-VAD-FMK), Annexin V-fluorescein isothiocyanate (FITC) and propidium iodide (PI) were purchased from BD Pharmingen (BD Biosciences, San Jose, CA, USA).

Cell culture and sanguinarine treatment. HLE B-3 cell line was obtained from the American Type Culture Collection (Manassas, VA, USA) and cultured in an incubator containing $5 \% \mathrm{CO}_{2}$ at $37^{\circ} \mathrm{C}$ in RPMI 1640 medium (cat. no. 21875091; Gibco; Thermo Fisher Scientific, Inc., Waltham, MA, USA) supplemented with $20 \%$ fetal bovine serum (Gibco; Thermo Fisher Scientific, Inc.), $100 \mu \mathrm{g} / \mathrm{ml}$ streptomycin and $100 \mathrm{U} / \mathrm{ml}$ penicillin (Gibco; Thermo Fisher Scientific, Inc.). Sanguinarine $(0.5 \mathrm{~g})$ was dissolved in $1 \mathrm{ml}$ DMSO and diluted into a $50 \mathrm{ml}$ stock solution with distilled water, which was used to prepare the testing concentrations. The experimental groups were treated with different concentrations of sanguinarine, and with or without pan-caspase inhibitor z-VAD-FMK or ROS inhibitor NAC. Z-VAD-FMK and NAC were dissolved in DMSO to obtain stock solutions of $50 \mathrm{mM}$ each, and added to $1 \mathrm{ml}$ culture wells at a final concentration of $50 \mu \mathrm{M}$. The control groups were treated with DMSO as vehicle.

MTT assay. HLE B-3 cells were seeded at a density of 4,000 cells/well in 96-well plates, and treated with 1, 2, 3 or $4 \mu \mathrm{M}$ sanguinarine. The control group was treated with the sanguinarine-free carrier solution (DMSO). After 24 or $48 \mathrm{~h}, 10 \mu 10.5 \%$ MTT solution was added to each well and further incubated for $4 \mathrm{~h}$. Subsequently, $100 \mu \mathrm{l}$ triple solution (10\% SDS, $5 \%$ isobutanol and $0.1 \% \mathrm{HCl}$ ) were added to each well to dissolve the formazan crystals and incubated overnight. The value of absorbance was measured at $570 \mathrm{~nm}$ using a microplate reader (Tecan Group, Ltd., Mannedorf, Switzerland). Cell proliferation was calculated as the percentage difference in the absorbance of treated cells compared with untreated cells (21). The anti-proliferative activity of sanguinarine was expressed as the half-maximal inhibitory concentration $\left(\mathrm{IC}_{50}\right)$ and the calculation formula was as follows: Inhibition $(\%)=\left[1-\left(\mathrm{OD}_{\text {observed }} / \mathrm{OD}_{\text {control }}\right)\right] \times 100 \%$.
Detection of apoptosis rate by flow cytometry. Flow cytometry was used to analyze the apoptosis of cells treated with $2 \mu \mathrm{M}$ sanguinarine. A total of $1 \times 10^{5}$ cells were harvested, washed with precooled PBS, and stained with Annexin V-FITC and propidium iodide (PI) for $15 \mathrm{~min}$, at room temperature and in the dark. The percentage of apoptotic cells was determined via flow cytometry (BD FACSAria ${ }^{\mathrm{TM}}$ III; BD Biosciences) and analyzed with BD CellQuest Pro ${ }^{\text {TM }}$ Software (version 5.1, BD Biosciences). Early stage apoptotic cells were stained only with Annexin V-FITC, and late stage apoptotic cells were stained with Annexin V-FITC and PI. Apoptotic cells were considered to be the sum of all early and late apoptotic cells.

Analysis of MMP. The mitochondria-specific fluorescent dye tetraethylbenzimidazolylcarbocyanine iodide (JC-1; Abcam, Cambridge, UK) was used to determine the alterations in the MMP of HLE B-3 cells treated with $2 \mu \mathrm{M}$ sanguinarine. Cells were harvested, washed and stained with $\mathrm{JC}-1$ for $30 \mathrm{~min}$ at $37^{\circ} \mathrm{C}$ in the dark, followed by washing and re-suspension with $300 \mu \mathrm{l}$ PBS. Variations in the MMP levels were measured at 527 and $590 \mathrm{~nm}$ by flow cytometry and data analyses were performed using Summit software (version 5.2, Beckman Coulter, Brea, CA, USA). In cases of mitochondrial damage, JC-1 will predominantly occur in its monomeric form in the cytoplasm and will emit green fluorescence, while in healthy cells, JC-1 forms aggregates and emits red fluorescence (22).

Caspase-3/7 activity assay. The activity of caspase-3/7 was detected by Caspase-GIo 3/7 Assay kit (Promega Corporation, Madison, WI, USA). An advantage of this kit is that it provides a luminogenic substrate of caspase-3/7 containing the tetrapeptide sequence DEVD, which has optimized caspase activity, luciferase activity and cell lysis. Following treatment of HLE B-3 cells with different concentrations of sanguinarine, with or without pan-caspase inhibitor z-VAD-FMK and ROS inhibitor NAC for $24 \mathrm{~h}, 100 \mu \mathrm{l}$ caspase-GIo $3 / 7$ reagent were added to each well of the 96 -well plates. Cells were incubated with the reagent for $1 \mathrm{~h}$, at $37^{\circ} \mathrm{C}$ and in the dark, and the luminescence signal was measured by a Luminometer (Tecan Group, Ltd.).

Detection of ROS. Following attachment, HLE B-3 cells were pretreated with $20 \mu \mathrm{M} 2^{\prime}, 7^{\prime}$-dichlorofluorescein diacetate (CM-H ${ }_{2}$ DCFDA; Invitrogen; Thermo Fisher Scientific, Inc.), a specific superoxide tracer dye, for $30 \mathrm{~min}$ at $37^{\circ} \mathrm{C}$ prior to testing. HLE B-3 cells treated with $2 \mu \mathrm{M}$ sanguinarine, with or without z-VAD-FMK, were incubated for $1 \mathrm{~h}$, harvested and re-suspended in PBS. Fluorescence was measured by flow cytometer with excitation/emission wavelengths of 488/525 nm and data analysis was performed as described above (23).

Western blot analysis. Following treatment with various concentrations of sanguinarine for different time intervals, HLE B-3 cells were lysed with radioimmunoprecipitation assay lysis buffer (Santa Cruz Biotechnology, Inc.) with protease and phosphatase inhibitors. The concentration of protein was determined using the Lowry-based Bio-Rad DC ${ }^{\mathrm{TM}}$ protein assay kit (Bio-Rad Laboratories, Inc., Hercules, CA, USA). Following denaturation, $30 \mu \mathrm{g}$ of proteins were loaded per lane and separated via 10\% SDS-PAGE. The separated proteins were transferred to a nitrocellulose membrane. The membranes were 
blocked with 5\% skimmed milk in TBS-Tween-20 for $1 \mathrm{~h}$ at room temperature. Primary antibodies against Bcl-2 (1:500; cat. no. sc-23960), Bax (1:500; cat. no. sc-20067), p38 (1:1,000; cat. no. 9212), JNK (1:1,000; cat. no. 9251), phospho-JNK (p-JNK; 1:1,000; cat. no. 9251), phospho-p38 (1:1,000; cat. no. 9211) and $\beta$-actin $(1: 1,000$; cat. no. 4967$)$ were incubated overnight at $4^{\circ} \mathrm{C}$. Following three 5 min washes with TBS-Tween-20, membranes were incubated with a horseradish peroxidase-labeled secondary antibody (1:5,000 dilution; cat. no. sc-2350; Santa Cruz Biotechnology, Inc.) for $1 \mathrm{~h}$ at room temperature, followed by enhanced chemiluminescent detection (Merck KGaA). $\beta$-actin was used as a loading control and for normalization. ImageJ Software version 1.46 (National Institute of Health, Bethesda, MA, USA) was used for densitometry analysis.

Statistical analysis. Data are expressed as the mean \pm standard deviation of individual groups. Differences between groups were adequately determined using one-way or two-way analysis of variance followed by Bonferroni post-hoc tests for multiple comparisons. $\mathrm{P}<0.05$ was considered to indicate a statistically significant difference. All statistical analyses were performed using GraphPad Prism software version 6 . (GraphPad Software, Inc., La Jolla, CA, USA).

\section{Results}

Sanguinarine reduces the proliferation of HLEs. To determine the effects of sanguinarine on the proliferation/viability of HLE B-3 cells, an MTT assay was performed. As exhibited in Fig. 1, the proliferation of HLE B-3 cells tended to decrease with increasing concentrations of sanguinarine and longer exposure times. The $\mathrm{IC}_{50}$ values of sanguinarine on HLE B-3 cells at 24 and $48 \mathrm{~h}$ were $3.60 \pm 0.68$ and $2.02 \pm 0.53 \mu \mathrm{M}$, respectively. At the same time points, compared with the control group, the proliferation of HLE B-3 cells decreased with increasing concentrations of sanguinarine. Similarly, at the same sanguinarine concentration, the proliferation of HLE B-3 cells was significantly decreased following longer treatments. Therefore, these results indicated that sanguinarine may have significantly inhibited the proliferation of HLE B-3 cells $(\mathrm{P}<0.01)$.

Sanguinarine inhibits the growth of HLEs by inducing cell apoptosis. To investigate the anti-proliferative mechanism of sanguinarine on HLEs, the apoptosis of HLE B-3 cells was evaluated using flow cytometry based on PI and Annexin V-FITC staining (Fig. 2). A significant number of HLE B-3 cells underwent apoptosis following treatment with $2 \mu \mathrm{M}$ sanguinarine for $24 \mathrm{~h}$. The apoptosis rate [the sum of late apoptosis (Q2) and early apoptosis (Q3)] of HLE B-3 cells was $30.00 \pm 2.36 \%$ following treatment with sanguinarine, while the apoptosis rate in the control group was $7.67 \pm 0.98 \%$ (Fig. 2). There was a significant difference between the two groups $(\mathrm{P}<0.01)$. These results demonstrated that treatment with sanguinarine induced apoptosis of HLEs, which may contribute to the inhibitory effect of sanguinarine on cell viability.

Sanguinarine induces apoptosis through a mitochondriadependent pathway. A study reported that mitochondrial dysfunction may be a critical mediator of apoptosis in cancer (24). To investigate whether mitochondria are involved in the

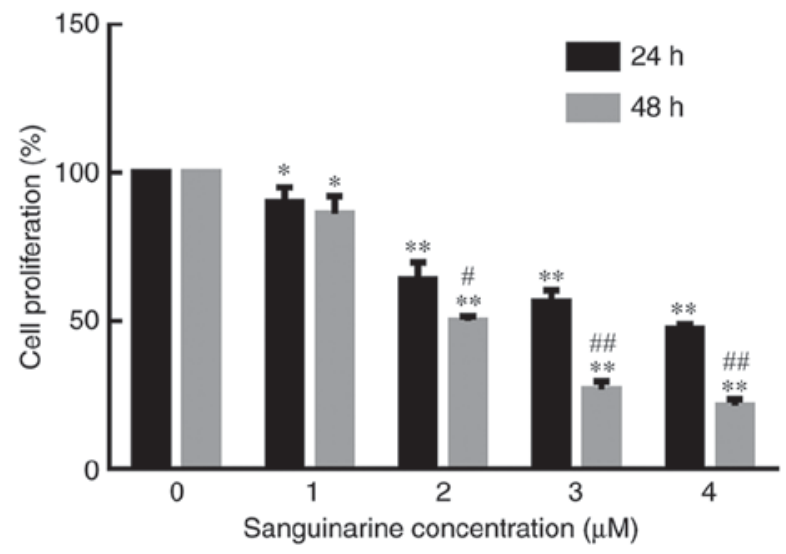

Figure 1. Sanguinarine has a significant anti-proliferative effect on HLE cells. The viability/proliferation of HLE cells treated with different concentrations of sanguinarine was evaluated at 24 and $48 \mathrm{~h}$ using an MTT assay $(\mathrm{n}=3)$. The results are expressed as the mean \pm standard deviation. ${ }^{*} \mathrm{P}<0.05$ and ${ }^{* *} \mathrm{P}<0.01$ vs. the control group ( $0 \mu \mathrm{M}$ of sanguinarine as a control group) ${ }^{\#} \mathrm{P}<0.05$ and ${ }^{\# \#} \mathrm{P}<0.01$ vs. $24 \mathrm{~h}$ (at the same concentrations of sanguinarine). HLE, human lens epithelial.

sanguinarine-induced apoptosis of HLEs, the MMP was analyzed by JC-1 staining. As exhibited in Fig. 3A, the percentage of cells which emitted red fluorescence was reduced in the mitochondria of HLE B-3 cells treated with sanguinarine, while the percentage of cells which emitted green fluorescence increased. These results indicated that MMP was significantly reduced following treatment of HLE B-3 cells with sanguinarine compared with the control group ( $\mathrm{P}<0.01$; Fig. $3 \mathrm{~A}$ and $\mathrm{B})$.

The process of apoptosis involving mitochondria is regulated via the ratio of Bcl-2 (anti-apoptotic protein) and Bax (pro-apoptotic protein), and a decrease in this ratio indicates the activation of the apoptotic pathway (25). This ratio is considered to be a key factor in controlling the integrity of mitochondrial membranes. Therefore, the protein levels of Bcl-2 and Bax were detected via western blotting. Subsequent to treatment with sanguinarine, the anti-apoptotic protein Bcl-2 was observed to be significantly decreased $(\mathrm{P}<0.01)$, while the pro-apoptotic protein Bax was increased $(\mathrm{P}<0.05)$. Therefore, the ratio of Bcl-2/Bax was decreased, which further supported the evidence that the mitochondria in HLE B-3 cells were damaged following treatment with sanguinarine (Fig. 3C). The flow cytometry and western blotting results suggested that sanguinarine may affect mitochondrial function and integrity and that mitochondria may be involved in sanguinarine-induced apoptosis.

Sanguinarine induces the activation of caspase in HLEs. Caspases 3 and 7 are considered key mediators of mitochondrial events of apoptosis, as their activation leads to the execution stage of apoptosis (26). In order to elucidate whether the activation of caspases was involved in sanguinarine-induced apoptosis, the activity of caspases 3 and 7 in control and treatment groups was analyzed using luminescent signal peaks proportional to the levels of caspase activity. The results demonstrated that sanguinarine stimulated caspase-3/7 activity in HLE B-3 cells in a concentration-dependent manner (Fig. 4A).

Subsequently, the pan-caspase inhibitor z-VAD-FMK and the ROS inhibitor NAC were used to investigate whether caspase activation and ROS generation, respectively, are 

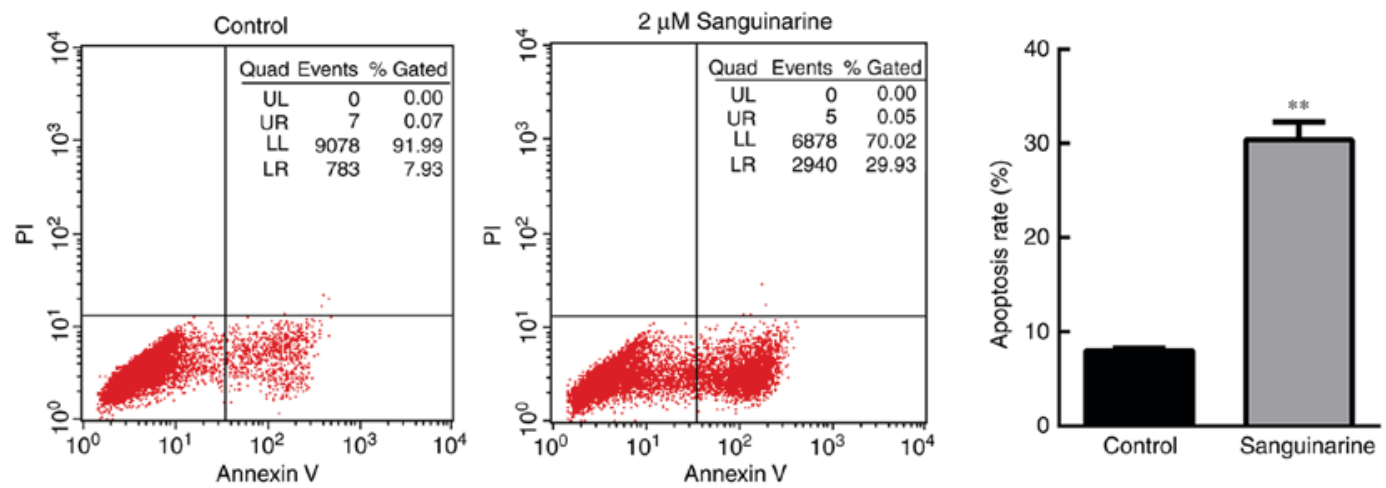

Figure 2. Sanguinarine induces apoptosis in HLE cells. Cell apoptosis of HLE B-3 cells was detected via flow cytometry ( $\mathrm{n}=3$ ). In the control group, $>90 \%$ of cells were gathered at the Q4 area, and $<10 \%$ of cells underwent apoptosis (Q2+Q3 areas). However, following treatment with $2 \mu \mathrm{M}$ sanguinarine for $24 \mathrm{~h}$, cells in the Q4 area decreased to 65.7\%, whereas cells in the Q2 and Q3 quadrants, which represent late and early apoptosis, respectively, were significantly increased. The results are expressed as the mean \pm standard deviation. ${ }^{* *} \mathrm{P}<0.01 \mathrm{vs}$. the control group. HLE, human lens epithelial; PI, propidium iodide.

A

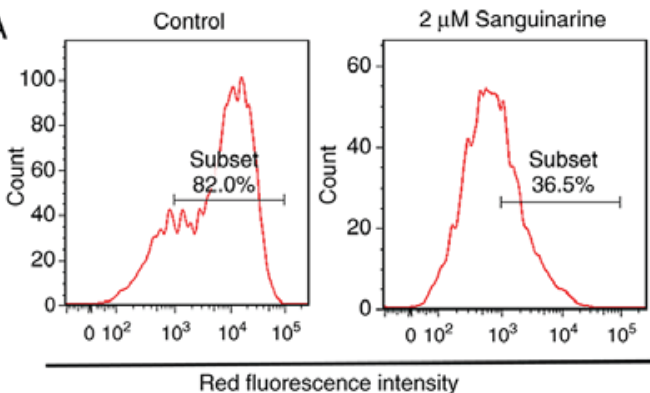

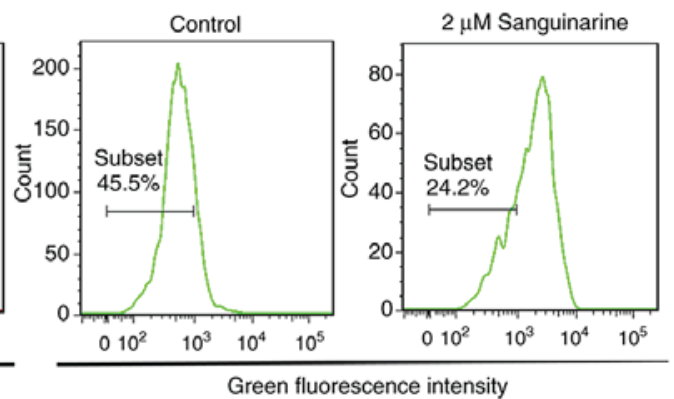

B

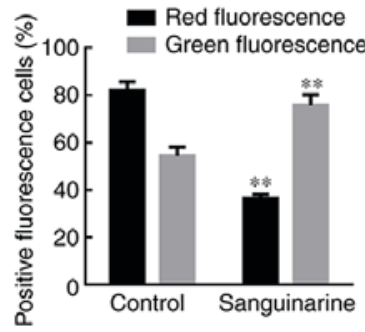

C
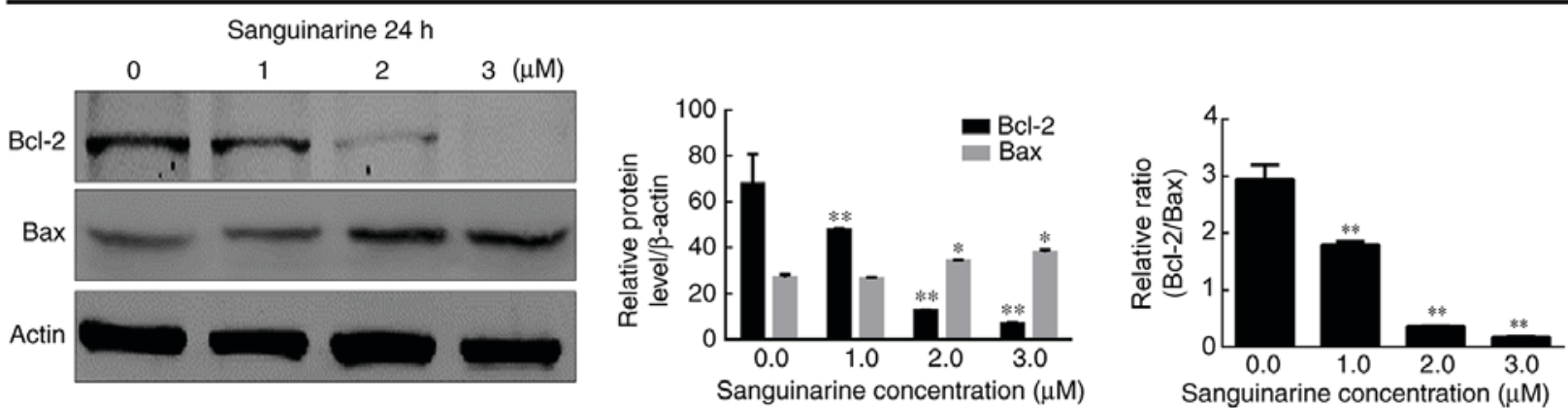

Figure 3. MMP and HLE cell integrity are reduced following treatment with sanguinarine. (A) JC-1 dye was used as an indicator of MMP. Compared with control group, the number of red fluorescence emitting cells, which is indicative of healthy mitochondria, was markedly decreased in the sanguinarine treatment group; however, the green fluorescence emitting cells, representing damaged mitochondria, were increased. (B) Quantification of types of fluorescence-emitting HLE cells obtained following staining with JC-1 $(\mathrm{n}=3)$. (C) The western blotting results demonstrated that the relative proportions of Bcl-2 and Bax, key regulators of mitochondrial membrane integrity, were significantly altered following treatment with sanguinarine $(n=3)$. The results are expressed as the mean \pm standard deviation. " $\mathrm{P}<0.05$ and ${ }^{* *} \mathrm{P}<0.01$ vs. respective control. HLE, human lens epithelial; JC-1, tetraethylbenzimidazolylcarbocyanine iodide dye; Bcl-2, B-cell lymphoma 2; Bax, apoptosis regulator BAX; MMP, mitochondrial membrane potential.

required for sanguinarine-induced apoptosis. The results of the flow cytometry analysis indicated that exposure to z-VAD-FMK or NAC significantly attenuated the effect of sanguinarine in inducing apoptosis compared with the single sanguinarine treatment group $(\mathrm{P}<0.01 ;$ Fig. $4 \mathrm{~B})$. These results demonstrated that $z$-VAD-FMK and NAC significantly reduced the levels of apoptosis induced by sanguinarine, and that sanguinarine-induced apoptosis may be caspase-dependent.

Sanguinarine-mediated ROS generation leads to apoptosis in HLEs. The association between ROS and mitochondria has been studied for a long time (27). A study has reported that the accumulation of ROS may be responsible for the mitochondrial dysfunction (28). Therefore, the association between the generation of ROS and mitochondrial dysfunction induced by sanguinarine was evaluated. CM-H2DCFDA was used to detect the amount of ROS in HLE B-3 cells. As presented in Fig. 5, following treatment with sanguinarine, flow cytometry data indicated that more cells exhibited the peak of fluorescence intensity (the peak shifted to the right), revealing that sanguinarine greatly increased the amount of ROS in HLE B-3 cells $(\mathrm{P}<0.01)$. To evaluate whether ROS generation serves an essential role in the process of apoptosis, the levels of ROS were determined following treatment with z-VAD-FMK and 
A

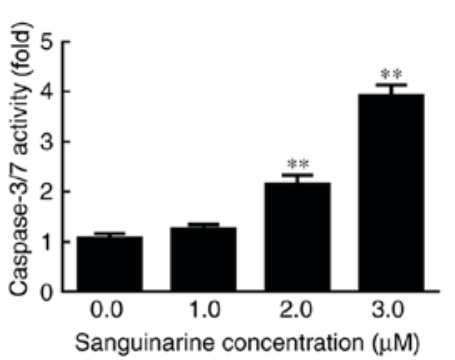

B
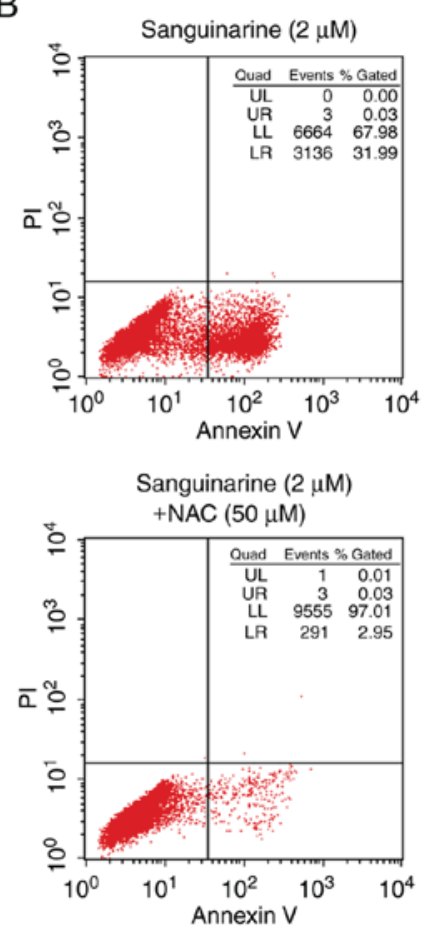
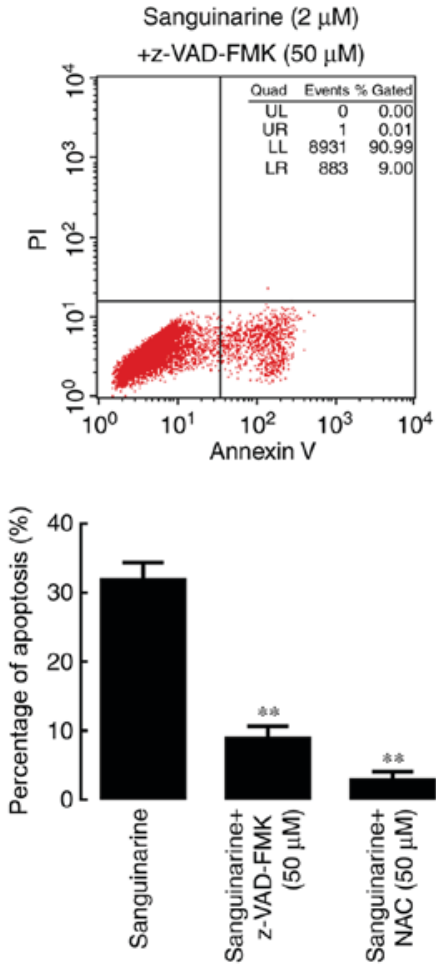

Figure 4. Caspase and ROS serve essential roles in sanguinarine-induced apoptosis in HLE cells. (A) Caspase-3/7 activity results demonstrated that following incubation with 1,2 or $3 \mu \mathrm{M}$ of sanguinarine for $24 \mathrm{~h}$, caspases $3 / 7$ activity levels were significantly increased (n=3). (B) In the sanguinarine only treatment group, 31\% of cells underwent apoptosis (Q2+Q3); however, in HLE B-3 cells co-treated with pan-caspase inhibitor z-VAD-FMK and sanguinarine, the apoptosis induced by sanguinarine was reduced; and cells co-treated with the ROS inhibitor NAC and sanguinarine also exhibited lower levels of apoptosis $(n=3)$. These results suggested that ROS generation and caspase activity were required for sanguinarine-induced apoptosis. The results are expressed as the mean \pm standard deviation. ${ }^{* *} \mathrm{P}<0.01$ vs. sanguinarine-only treatment. HLE, human lens epithelial; ROS, reactive oxygen species; $z-V A D-F M K$, benzyloxycarbonyl-Val-Ala-Asp (OMe) fluoromethylketone; NAC, N-acetylcysteine; PI, propidium iodide.
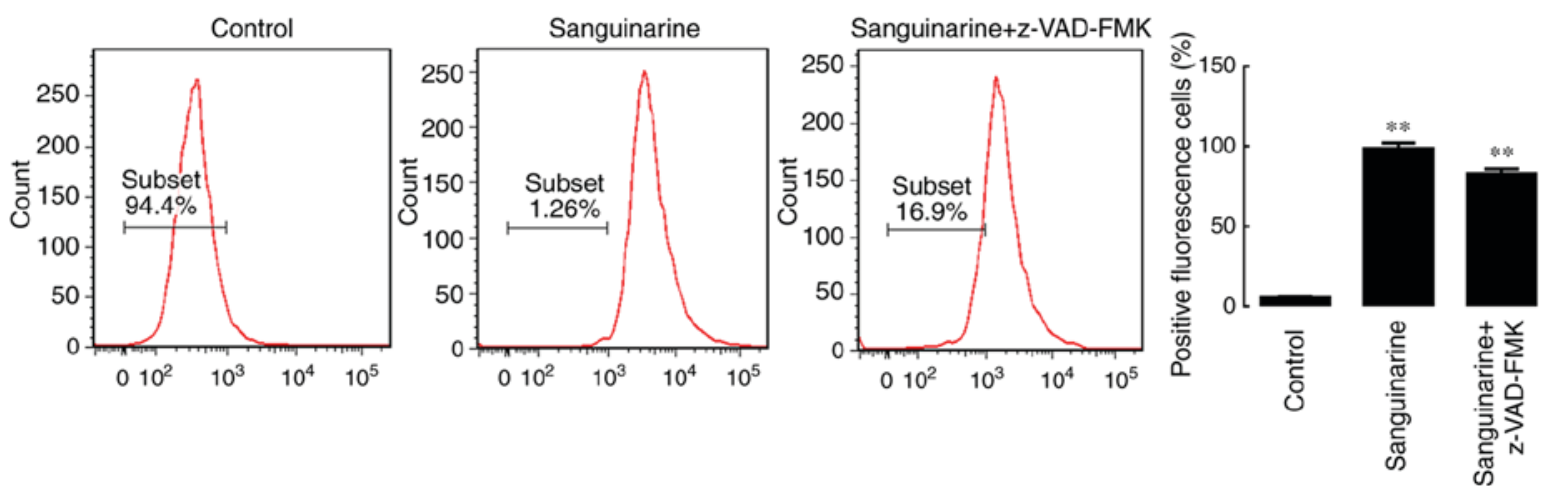

Figure 5. ROS generation is the initial step in sanguinarine-induced apoptosis in human lens epithelial cells. The data in the distribution plots represents the percentage of ROS-negative cells, and the bar graph indicate the percentage of ROS-positive cells, calculated by subtracting the percentage of ROS-negative cells to the total number of cells. The results are expressed as the mean \pm standard deviation. ${ }^{* *} \mathrm{P}<0.01$ vs. sanguinarine-only treatment. ROS, reactive oxygen species; z-VAD-FMK, benzyloxycarbonyl-Val-Ala-Asp (OMe) fluoromethylketone.

sanguinarine. This treatment, however, did not prevent the generation of ROS, suggesting that ROS generation may be an upstream step in sanguinarine-induced apoptosis.

Sanguinarine induces apoptosis of HLEs by activating the MAPK pathway. The MAPK pathway has been demonstrated to be closely associated with the process of apoptosis (29). To test whether the MAPK pathway serves a similar role in sanguinarine-induced apoptosis, HLE B-3 cells were treated with different concentrations of sanguinarine. As presented in Fig. 6A, treatment with sanguinarine promoted the phosphorylation of JNK and p38 in a dose-dependent manner. Moreover, longer treatments with $2 \mu \mathrm{M}$ sanguinarine $(2,4$ and $6 \mathrm{~h})$ significantly up-regulated the expression of p-JNK and p-P38 compared with the control group (Fig. 6B). Among these exposure times, the $2 \mathrm{~h}$ treatment resulted in higher levels of phosphorylation. To further determine whether the activation of JNK and p38 is a key step in sanguinarine-induced apoptosis, HLE B-3 cells were exposed to sanguinarine with or without ROS inhibitor NAC to assess whether reduced ROS generation 

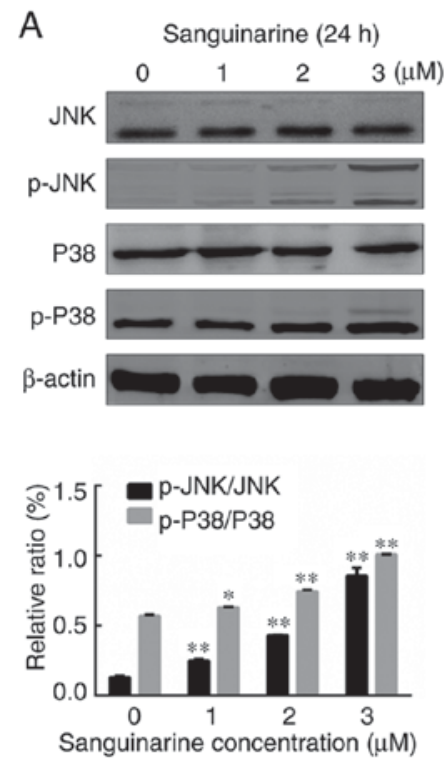
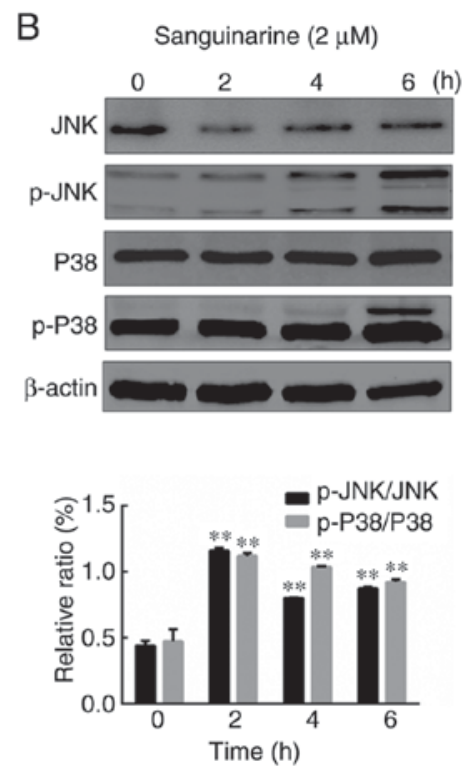
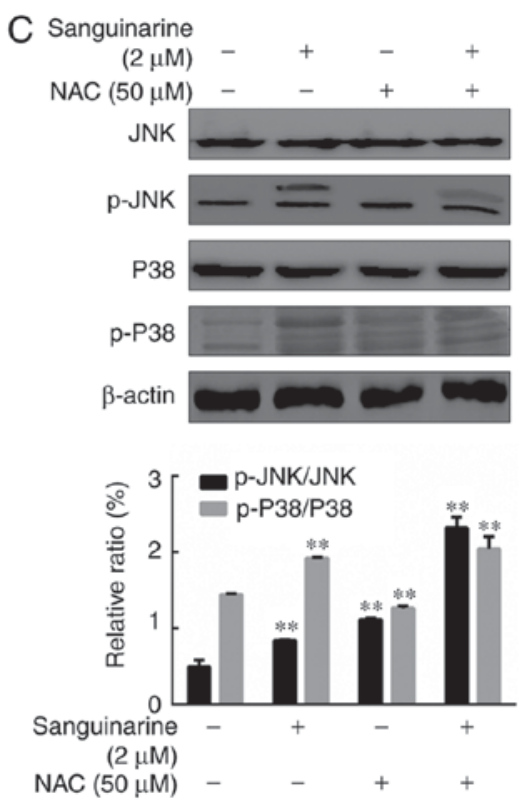

Figure 6. MAPK pathway is involved, but serves a subsidiary role in sanguinarine-induced apoptosis in human lens epithelial cells. The effect of different concentrations of sanguinarine (A) and different time treatments (B) on the activation of MAPK pathway-related proteins (JNK, p-JNK, P38 and p-P38) was detected using western blotting $(\mathrm{n}=3)$. The western blotting results demonstrated that both JNK and p38 were activated by sanguinarine in a dose-dependent manner ( $n=3)$. (C) NAC, a specific ROS inhibitor, was applied in a co-treatment with sanguinarine to determine whether it affected the activation of MAPK pathway components observed following treatment with sanguinarine $(n=3)$. Compared with sanguinarine single treatment group, co-treatment with sanguinarine and ROS inhibitor NAC significantly increased the levels of p-JNK and p-P38. The results are expressed as the mean \pm standard deviation. ${ }^{*} \mathrm{P}<0.05$ and ${ }^{* *} \mathrm{P}<0.01$. MAPK, mitogen-activated protein kinase; ROS, reactive oxygen species; NAC, N-acetylcysteine; p, phosphorylated; JNK, c-Jun N-terminal kinase.

prevents the phosphorylation of p38 and JNK. Co-treatment of HLE B-3 cells with sanguinarine and ROS inhibitor NAC significantly increased the expression levels of p-JNK and p-P38 compared with sanguinarine or NAC alone (Fig. 6C). These results suggested that the MAPK pathway is associated with sanguinarine-induced apoptosis, but it is not the primary target of sanguinarine.

\section{Discussion}

Over 8 million cataract operations are performed every year in Europe and the United States, and the number is even higher in China (30). PCO is the most common complication encountered following cataract surgery, and it seriously affects the quality of life of the patients through secondary loss of vision (2). The development of PCO is affected by various factors, but the proliferation and migration of HLEs is a common cause of PCO (31). Discovering novel agents that may effectively suppress the proliferation of HLEs and prevent $\mathrm{PCO}$ is of the utmost importance.

In the present study, it was verified that sanguinarine significantly reduced the viability of HLE B-3 cells in a doseand time-dependently manner, and that it induced apoptosis. The apoptosis effects were possibly mediated by early ROS generation, which may promote the activation of p38 and JNK, leading to the activation of the caspase pathway and causing apoptosis. Furthermore, the results seem to suggest that ROS generation may be essential for sanguinarine-mediated apoptosis. Thus, sanguinarine may be a potential therapeutic agent in the treatment of patients with PCO in the future.

ROS, the by-products of ATP production, are primarily formed by the interaction of oxygen molecules with electrons escaping from the mitochondrial respiratory chain (32). Normally, there is an antioxidant system that controls the basal levels of ROS as, in excess, these may cause cellular damage by reacting with DNA, proteins, lipids and even the electron transport chain itself $(33,34)$. Therefore, ROS generation is closely associated with mitochondrial function and cell health. Reports have also demonstrated that elevated levels of basal ROS in cancer cells are conducive to increased cell proliferation (35). Thus, ROS have complex roles within the scope of biological processes that occur in cells, making it difficult to understand how levels of oxidants are involved in cell growth and maintenance and how ROS may be used therapeutically (35). The present results seem to suggest that treatment with sanguinarine may have significantly increased the generation of ROS in HLE B-3 cells, and inhibiting its generation may have reduced the levels of apoptosis observed in these cells.

As mentioned previously, upregulation of ROS in cells may result in serious mitochondrial and cellular damage $(36,37)$. In the present study, the MMP of HLE B-3 cells treated with sanguinarine was significantly reduced compared with the control group. The pro-apoptotic protein Bax was also upregulated, while the anti-apoptotic protein $\mathrm{Bcl}-2$ was downregulated. This alteration in the $\mathrm{Bax} / \mathrm{Bcl}-2$ ratio is an indicator of mitochondrial membrane damage $(38,39)$. Once mitochondria are damaged, cytochrome $\mathrm{c}$ is released and forms a complex with apoptosis protease-activating factor-1 to further activate caspase-9, which in turn activates caspases 3 and 7 (40-42). The present study indicated that the activation of caspases is involved in sanguinarine-induced apoptosis, as the use of pan-caspase inhibitor z-VAD-FMK resulted in the reduction of apoptosis levels following treatment with sanguinarine. 
Therefore, sanguinarine may have induced apoptosis through caspase-associated pathways.

Finally, the involvement of the MAPK pathway, which is closely associated with cell proliferation and survival $(43,44)$, was evaluated. Sanguinarine promoted the phosphorylation of JNK and p38 kinases in HLE B-3 cells, suggesting that MAPK signaling pathway may be involved in sanguinarine-induced apoptosis.

In conclusion, sanguinarine may significantly affect the viability of HLE B-3 cells by inducing apoptosis. ROS generation and caspase activation may serve as key mediators of sanguinarine-induced apoptosis. The MAPK signaling pathway may be involved. Sanguinarine may possibly be used as a potential drug for preventing PCO or even the development of cataracts in the future. However, the present study is only preliminary. Further in vivo research and clinical studies should be undertaken to verify the potential beneficial effects of sanguinarine on PCO treatment.

\section{Acknowledgements}

Not applicable.

\section{Funding}

The present study was supported by The National Natural Science Funds (grant no. 81500705).

\section{Availability of data and materials}

All data generated or analyzed during this study are included in this published article.

\section{Authors' contributions}

YZ and WRH contributed equally to the present study. YZ and WRH designed the study, performed the experimental work, collected, analyzed and interpreted the data, and drafted and revised the manuscript. All authors read and approved the final manuscript.

\section{Ethics approval and consent to participate}

Not applicable.

\section{Patient consent for publication}

Not applicable.

\section{Competing interests}

The authors declare that they have no competing interests.

\section{References}

1. Chang KC and Petrash JM: Aldose reductase mediates transforming growth factor $\beta 2$ (TGF- $\beta 2$ )-induced migration and epithelial-to-mesenchymal transition of lens-derived epithelial cells. Invest Ophthalmol Vis Sci 56: 4198-4210, 2015.

2. Meacock WR, Spalton DJ, Boyce J and Marshall J: The effect of posterior capsule opacification on visual function. Invest Ophthalmol Vis Sci 44: 4665-4669, 2003.
3. Fernandez V, Fragoso MA, Billotte C, Lamar P, Orozco MA, Dubovy S, Willcox M and Parel JM: Efficacy of various drugs in the prevention of posterior capsule opacification: Experimental study of rabbit eyes. J Cataract Refract Surg 30: 2598-2605, 2004.

4. Jiang YX, Lu Y, Liu TJ, Yang J, Chen Y and Fang YW: Using HSV-TK/GCV suicide gene therapy to inhibit lens epithelial cell proliferation for treatment of posterior capsular opacification. Mol Vis 17: 291-299, 2011.

5. Rönbeck M, Zetterström C, Wejde G and Kugelberg M: Comparison of posterior capsule opacification development with 3 intraocular lens types: Five-year prospective study. J Cataract Refract Surg 35: 1935-1940, 2009.

6. Huang WR, Zhang Y and Tang X: Shikonin inhibits the proliferation of human lens epithelial cells by inducing apoptosis through ROS and caspase-dependent pathway. Molecules 19: 7785-7797, 2014.

7. Laster LL and Lobene RR: New perspectives on Sanguinaria clinicals: Individual toothpaste and oral rinse testing. J Can Dent Assoc 56 (Suppl 7): S19-S30, 1990.

8. Miao F, Yang XJ, Zhou L, Hu HJ, Zheng F, Ding XD, Sun DM, Zhou CD and Sun W: Structural modification of sanguinarine and chelerythrine and their antibacterial activity. Nat Prod Res 25: 863-875, 2011.

9. Slaninová I, Pěnčíková K, Urbanová J, Slanina J and Táborská E: Antitumour activities of sanguinarine and related alkaloids. Phytochem Rev 13: 51-68, 2014.

10. Gaziano R, Moroni G, Buè C, Miele MT, Sinibaldi-Vallebona P and Pica F: Antitumor effects of the benzophenanthridine alkaloid sanguinarine: Evidence and perspectives. World J Gastrointest Oncol 8: 30-39, 2016.

11. Godowski KC: Antimicrobial action of sanguinarine. J Clin Den 1: 96-101, 1989

12. Achkar IW, Mraiche F, Mohammad RM and Uddin S: Anticancer potential of sanguinarine for various human malignancies. Future Med Chem 9: 933-950, 2017.

13. Wang Q, Dai P, Bao H, Liang P, Wang W, Xing A and Sun J: Anti-inflammatory and neuroprotective effects of sanguinarine following cerebral ischemia in rats. Exp Ther Med 13: 263-268, 2017.

14. Kalogris C, Garulli C, Pietrella L, Gambini V, Pucciarelli S, Lucci C, Tilio M, Zabaleta ME, Bartolacci C, Andreani C, et al: Sanguinarine suppresses basal-like breast cancer growth through dihydrofolate reductase inhibition. Biochem Pharmacol 90: 226-234, 2014

15. Kuttikrishnan S, Siveen KS, Prabhu KS, Khan AQ, Akhtar S, Mateo JM, Merhi M, Taha R, Omri HE, Mraiche F, et al: Sanguinarine suppresses growth and induces apoptosis in childhood acute lymphoblastic leukemia. Leuk Lymphoma 6: 1-13, 2018.

16. Gong X, Chen Z, Han Q, Chen C, Jing L, Liu Y, Zhao L, Yao X and Sun $X$ : Sanguinarine triggers intrinsic apoptosis to suppress colorectal cancer grow th through disassociation between STRAP and MELK. BMC Cancer 18: 578, 2018.

17. Vogt A, Tamewitz A, Skoko J, Sikorski RP, Giuliano KA and Lazo JS: The benzo[c]phenanthridine alkaloid, sanguinarine, is a selective, cell-active inhibitor of mitogen-activated protein kinase phosphatase-1. J Biol Chem 280: 19078-19086, 2005.

18. Lee TK, Park C, Jeong SJ, Jeong MJ, Kim GY, Kim WJ and Choi YH: Sanguinarine induces apoptosis of human oral squamous cell carcinoma KB cells via inactivation of the PI3K/Akt signaling pathway. Drug Dev Res 77: 227-240, 2016.

19. Yang J, Fang Z, Wu J, Yin X, Fang Y, Zhao F, Zhu S and Li Y: Construction and application of a lung cancer stem cell model: Antitumor drug screening and molecular mechanism of the inhibitory effects of sanguinarine. Tumour Biol 37: 13871-13883, 2016.

20. Meng YY, Liu Y, Hu ZF, Zhang Y, Ni J, Ma ZG, Liao HH, Wu QQ and Tang QZ: Sanguinarine attenuates lipopolysaccharide-induced inflammation and apoptosis by inhibiting the TLR4/NF- $\mathrm{B}$ pathway in $\mathrm{H} 9 \mathrm{c} 2$ cardiomyocytes. Curr Med Sci 38: 204-211, 2018.

21. Nguyen KC, Willmore WG and Tayabali AF: Cadmium telluride quantum dots cause oxidative stress leading to extrinsic and intrinsic apoptosis in hepatocellular carcinoma HepG2 cells. Toxicology 306: 114-123, 2013.

22. Zhang DS, Li YY, Chen XJ, Li YJ, Liu ZY, Xie WJ and Sun ZL: BCL2 promotor methylation and miR-15a/16-1 upregulation is associated with sanguinarine-induced apoptotic death in rat HSC-T6 cells. J Pharmacol Sci 127: 135-144, 2015. 
23. He D, Ma X, Chen Y, Cai Y, Ru X, Bruce IC, Xia Q, Shi G and Jin J: Luteolin inhibits pyrogallol-induced apoptosis through the extracellular signal-regulated kinase signaling pathway. FEBS J 279: 1834-1843, 2012.

24. Hsu PC, Huang YT, Tsai ML, Wang YJ, Lin JK and Pan MH: Induction of apoptosis by shikonin through coordinative modulation of the Bcl-2 family, p27, and p53, release of cytochrome c, and sequential activation of caspases in human colorectal carcinoma cells. J Agric Food Chem 52: 6330-6337, 2004

25. Pradelli LA, Bénéteau M and Ricci JE: Mitochondrial control of caspase-dependent and -independent cell death. Cell Mol Life Sci 67: 1589-1597, 2010.

26. Lakhani SA, Masud A, Kuida K, Porter GA Jr, Booth CJ, Mehal WZ, Inayat I and Flavell RA: Caspases 3 and 7: Key mediators of mitochondrial events of apoptosis. Science 311: 847-851, 2006

27. Zorov DB, Juhaszova M and Sollott SJ: Mitochondrial reactive oxygen species (ROS) and ROS-induced ROS release. Physio Rev 94: 909-950, 2014.

28. Fonseca-Silva F, Inacio JD, Canto-Cavalheiro MM and Almeida-Amaral EE: Reactive oxygen species production and mitochondrial dysfunction contribute to quercetin induced death in leishmania amazonensis. PLoS One 6: e14666, 2011.

29. Sui X, Kong N, Ye L, Han W, Zhou J, Zhang Q, He C and Pan H: p38 and JNK MAPK pathways control the balance of apoptosis and autophagy in response to chemotherapeutic agents. Cancer Lett 344: 174-179, 2014

30. Wang SY, Stem MS, Oren G, Shtein R and Lichter PR: Patient-centered and visual quality outcomes of premium cataract surgery: A systematic review. Eur J Ophthalmol 27: 387-401, 2017.

31. Awasthi N, Guo S and Wagner BJ: Posterior capsular opacification: A problem reduced but not yet eradicated. Arch Ophthalmol 127: 555-562, 2009.

32. Poyton RO, Ball KA and Castello PR: Mitochondrial generation of free radicals and hypoxic signaling. Trends Endocrinol Metab 20: 332-340, 2009.

33. Ricci JE, Gottlieb RA and Green DR: Caspase-mediated loss of mitochondrial function and generation of reactive oxygen species during apoptosis. J Cell Biol 160: 65-75, 2003.

34. Park SY, Kim DY, Kang JK, Park G and Choi YW: Involvement of activation of the Nrf2/ARE pathway in protection against 6-OHDA-induced SH-SY5Y cell death by $\alpha$-iso-cubebenol. Neurotoxicology 44: 160-168, 2014.

35. Schumacker PT: Reactive oxygen species in cancer cells: Live by the sword, die by the sword. Cancer Cell 10: 175-176, 2006.
36. Zhang B, Xie QY, Quan Y, Pan XM and Liao DF: Reactive oxygen species induce cell death via Akt signaling in rat osteoblast-like cell line ROS 17/2.8. Toxicol Ind Health 31: 1236-1242, 2013

37. Zubair H, Khan HY, Sohail A, Azim S, Ullah MF, Ahmad A, Sarkar FH and Hadi SM: Redox cycling of endogenous copper by thymoquinone leads to ROS-mediated DNA breakage and consequent cell death: Putative anticancer mechanism of antioxidants. Cell Death Dis 4: e660, 2013.

38. Jeong $\mathrm{SY}$ and Seol DW: The role of mitochondria in apoptosis. BMB Rep 41: 11-22, 2008.

39. Kocic G, Tomovic K, Kocic H, Sokolovic D, Djordjevic B, Stojanovic S, Arsic I and Smelcerovic A: Antioxidative, membrane protective and antiapoptotic effects of melatonin, in silico study of physico-chemical profile and efficiency of nanoliposome delivery compared to betaine. RSC Adv 7: 1271-1281, 2017.

40. Zhou J and Tang XC: Huperzine A attenuates apoptosis and mitochondria-dependent caspase- 3 in rat cortical neurons. FEBS Lett 526: 21-25, 2002

41. Yuyama K, Yamamoto H, Nishizaki I, Kato T, Sora I and Yamamoto T: Caspase-independent cell death by low concentrations of nitric oxide in PC12 cells: Involvement of cytochrome $\mathrm{C}$ oxidase inhibition and the production of reactive oxygen species in mitochondria. J Neurosci Res 73: 351-363, 2003.

42. Zhu JJ, Xu YQ, He JH, Yu HP, Huang CJ, Gao JM, Dong QX, Xuan YX and Li CQ: Human cardiotoxic drugs delivered by soaking and microinjection induce cardiovascular toxicity in zebrafish. J Appl Toxicol 34: 139-148, 2014.

43. Chao JI, Su WC and Liu HF: Baicalein induces cancer cell death and proliferation retardation by the inhibition of $\mathrm{CDC} 2$ kinase and survivin associated with opposite role of p38 mitogen-activated protein kinase and AKT. Mol Cancer Ther 6: 3039-3048, 2007.

44. He XQ, Chen R, Yang P, Li AP, Zhou JW and Liu QZ: Biphasic effect of arsenite on cell proliferation and apoptosis is associated with the activation of JNK and ERK1/2 in human embryo lung fibroblast cells. Toxicol Appl Pharmacol 220: 18-24, 2007.

This work is licensed under a Creative Commons

Attribution-NonCommercial-NoDerivatives 4.0 International (CC BY-NC-ND 4.0) License. 